\title{
Single Step Deposition of an Interacting Layer of Perovskite Matrix with Embedded Quantum Dots.
}

\author{
ThiTuyen Ngo, ${ }^{1}$ Isaac Suarez, ${ }^{2}$ Rafael S. Sanchez, ${ }^{1}$ Juan P. Martinez-Pastor, ${ }^{2}$ and \\ Ivan Mora Sero ${ }^{1, *}$ \\ ${ }^{1}$ Institute of Advanced Materials (INAM), UniversitatJaume I, 12006 Castelló, \\ Spain. \\ ${ }^{2}$ UMDO, Instituto de Ciencia de los Materiales, Universidad de Valencia, 46071 \\ Valencia, Spain. \\ *Corresponding authors: sero@uji.es
}

\begin{abstract}
.
Hybrid lead halide perovskite derivatives (PS) have emerged as very promising materials for the development of optoelectronic devices in the last few years. At the same time, inorganic nanocrystals with quantum confinement (QDs) possess unique properties that make them suitable materials for the development of photovoltaics, imaging and lighting applications, among others. In this work, we report on a new methodology for the deposition of high quality, large grain size and pinhole free PS films $\left(\mathrm{CH}_{3} \mathrm{NH}_{3} \mathrm{PbI}_{3}\right)$ with embedded $\mathrm{PbS}$ and $\mathrm{PbS} / \mathrm{CdS}$ core/shell Quantum Dots (QDs). The strong interaction between both semiconductors is revealed by the formation of an exciplex state, which is monitored by photoluminescence and electroluminescence experiments. The radiative exciplex relaxation is centered in the near infrared region (NIR), $\approx 1200$ $\mathrm{nm}$, which correspond to lower energies than the corresponding band gap of both perovskite (PS) and QDs themselves. Our approach allows fabricating multiwavelength light emitting diodes (LEDs) based on a PS matrix with embedded QDs, which show considerably low turn-on potentials. The presence of the
\end{abstract}


exciplex state of PS and QDs opens up a broad range of possibilities with important implications in both LEDs and solar cells.

\section{TOC Figure:}

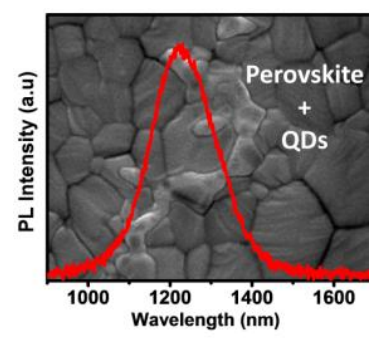

High quality pinhole free perovskite halide thin layer exhibiting IR exciplex state emission have been prepared introducing $\mathrm{Pb} / \mathrm{CdS}$ QDs in single step deposition process. 
Nowadays, the research focused on the development and applicability of hybrid lead halide perovskite materials (PS) probably constitutes the hottest topic in the photovoltaics field due to several reasons. Firstly,certified photoconversion efficiencies as high as $22.1 \%$ have been reported so far. ${ }^{1}$ Moreover, the impressive rise in the conversion efficiencies has been reached only few years after the first reports on PS based liquid electrolyte solar cells in 2009. ${ }^{2}$ Very importantly, it has been demonstrated that PS shows reduced non-radiative recombination pathways; this fact has significantly contributed to reachhigh photoconversion efficiencies. ${ }^{3}$, ${ }^{4}$ Additionally, the application of PS derivatives for the development of light emitting devices, ${ }^{5}$ as LEDs, ${ }^{6-8}$ light amplifiers ${ }^{9}$ or lasers, ${ }^{10-12}$ has been already demonstrated, which is a consequence of the outstanding properties of these materials. It is worth pointing out that PS can be prepared from solution methods at low processing temperatures and, consequently, using low cost fabrication techniques. Furthermore, PS can be easily combined with other materials as colloidal Quantum Dots (QDs), ${ }^{13},{ }^{14}$ which can induce interesting synergies, thus giving rise to new properties or phenomena that do not exist for the single materials themselves.

Colloidal QDs constitute a very interesting family of nanomaterials that have been extensively studied for optoelectronic applications during the last two decades due to their size-tunable bandgap, relatively facile synthesis, high monodispersity, and the possibility of being processed in solution. ${ }^{15-18}$ Despites the high potentiality of developing advanced optoelectronic systems by exploiting the combination of PS and QDs, there are only few examples in the literature reporting on these issues; PbS QDs combined with PS has been the most general system studied so far. Particularly, precedents in the literature report on the utilization of PS as a capping agent of QDs, thus providing QD/PS core/shell structures, ${ }^{19-21}$ which have shown outstanding efficiencies, close to $9 \%$, in colloidal QD solar cell configuration. ${ }^{19} \mathrm{PbS}$ QDs have been deposited on perovskite layers for photovoltaic applications, which enhances the light harvesting at IR wavelengths and therefore, the photogenerated short-circuit 
current $\left(J_{s c}\right){ }^{22,}{ }^{23}$ However a more intimate interaction between PS and QDs has been obtained by the preparation of layers that consists of a PS matrix with embedded QDs using two-step spin coating method. ${ }^{13}$ This approach has an enormous potentiality for the development of different optoelectronic devices. Highly efficient QD based near-infrared light-emitting diodes have been obtained by combining a PS matrix with PbS QDs with good lattice matching that forms a type I junction. ${ }^{24}$ In addition, PbS QDs have been also used as efficient seeds for the preparation high-performance planar heterojunction perovskite solar cells. ${ }^{25}$ We have recently analyzed the interaction between PS and PbS/CdS core-shell QDs bilayers that form a type II junction. ${ }^{14}$ More specifically, we reported on the identification of an exciplex emission that results from the strong interaction between both semiconductors films, by means of optical pumping and electrical injection at energies lower than the bandgap of the single materials themselves. As a continuation of our previous work, we envisaged that this effect could be much more pronounced if the QDs were inserted into the PS films instead of stacking layers of the corresponding single materials. Therefore, in the present paper we propose a novel one step spin coating method to produce a nanocomposite that consists of either core $\mathrm{PbS}$ or core/shell $\mathrm{PbS} / \mathrm{CdS}$ QDs embedded into a PS matrix. This method permits to obtain very uniform PS-QDs layers, with significantly larger PS grain size, compared to bare PS (reference sample), which clearly exhibit exciplex emission.

$\mathrm{PbS} / \mathrm{CdS}$ core/shell QDs were generally synthesized using oleic acid (OA) that controls the dimension of QDs and prevents their aggregation. However OAcapped QDs are not soluble in dimethylformamide (DMF), which is the commonly employed solvent to dissolve th e PS precursor, and therefore a ligand exchange treatment becomes necessary to overcome this solubility limitation. Fig. 1a shows the ligand exchange processes. Core/shell $\mathrm{PbS} / \mathrm{CdS}$ QDs capped with OA ligands were originally dispersed in octane, and mixed with a DMF solution containing PS precursors $\left(\mathrm{PbI}_{2}\right.$ and $\left.\mathrm{CH}_{3} \mathrm{NH}_{3} \mathrm{I}\right)$. In this step, two separated phases were detected: QDs dispersed in octane on the top and PS dissolved in DMF at the 
bottom (Bottle1). After stirring the mixed solution, OA is replaced by a $\mathrm{CH}_{3} \mathrm{NH}_{3} \mathrm{PbI}_{3}$ PS capping and QDs were transferred into DMF phase with perovskite precursors (dark phase, Bottle2). PS capped QDs were precipitated with toluene, dried and finally dispersed in a solution containing the PS precursors (PS-QDs solution, Bottle3) that will be used for the spin coating deposition of the PS with embedded QDs films (see the details in the experimental part see Supporting Information). The absorbance and photoluminescence (PL) in solution of PS capped QDs is red shifted (see Fig. S1a and S1b) and the PL quantum yield is significantly reduced after ligand exchange, from $18 \%$ to $0.9 \%$ see Fig. S1b.

(a)

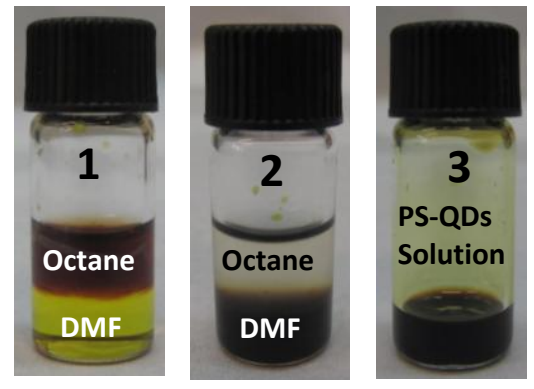

(b)

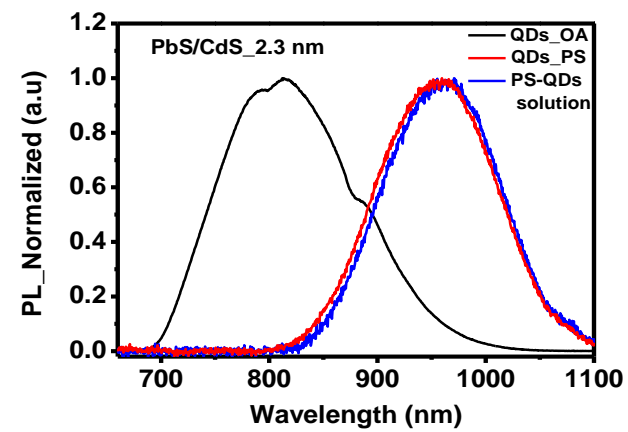

Fig 1. (a)Pictures of the different phases of QD ligand exchange process. Bottle 1: two phase solution formed by DMF with perovskite precursors at the bottom and PbS/CdS core/shell QDs capped with OA solved in octane on the top. Bottle 2: after 30 minutes stirring, the QDs were transferred into DMF since OA was exchanged by a PS shell. Bottle 3: PS capped QDs were precipitated with toluene, dried and finally dispersed in a DMF solution containing PS precursors $\left(\mathrm{PbI}_{2}\right.$ and $\mathrm{CH}_{3} \mathrm{NH}_{3} \mathrm{I}$, 1:1 molar ratio); this solution is used for the deposition of perovskite layer with embedded QDs. (b)Photoluminescence spectra of PbS/CdS QDs in solution before (OA capped), after ligand exchange (PS capped) and the final PS-QDs solutions $\left(\lambda_{\text {excitation }}=650 \mathrm{~nm}\right.$ ).

Fig. 1b shows the PL spectra of the QDs solutions before (OA capped) and after ligand exchange (PS capped). The PL peak after ligand exchange was red shifted depending on the QD size, being150 $\mathrm{nm}$ for QDs of $2.3 \mathrm{nmin}$ diameter (from $812 \mathrm{~nm}$ to $962 \mathrm{~nm}$, Fig $1 \mathrm{~b}$ and Fig S1c) and $83 \mathrm{~nm}$ for QDs of $3 \mathrm{~nm}$ in diameter (from $893 \mathrm{~nm}$ to $976 \mathrm{~nm}$, Fig. S1d), thus indicating that this effect is more significant for the smaller sized QDs, which is in good correlation with 
previous reports. ${ }^{20}$ PS capping allows the wave function to spread out of QDs, thus reducing the quantum confinement that provokes the strong red-shift. The PL emission of the QD/PS nanocrystals dispersed in the PS solution (PS-QDs solution) did not vary compared to that resulting from dispersing QD/PS in DMF, see Fig. 1b, S1c and S1d. This fact indicates that PS shell does not dissociated into the corresponding precursors or does not suffer from chemical variations after being dispersed in DMF, which demonstrates the robustness of the PS shell. The QY of PS-QDs solution was calculated as a function of QD concentration, which decreases linearly from $1.6 \%$ to $1 \%$ when QD concentration was increased from 200 to $400 \mathrm{mg} / \mathrm{ml}$ (Fig. S2).

PS and PS-QDs films were spin coated onto compact $\mathrm{TiO}_{2}$ at $9000 \mathrm{rpm}$ from the PS and PS-QDs solutions, respectively inside a glove box. The films were annealed at $65^{\circ} \mathrm{C}$ for 1 minute and subsequently at $100^{\circ} \mathrm{C}$ during 2 minutes. Very homogenous, pinhole free morphology was obtained for both PS and PSQDs films (Fig. 2a and 2b respectively). The introduction of QDs in the solution containing the perovskite precursors has a dramatic effect of on the grain size, which is increased in 4-5 fold factor. These results point to the significant role of the QDs on the nucleation of the PS crystals,as it has been recently reported. ${ }^{25}$ The increase of grain size produced a slight red shift, around $4 \mathrm{~nm}$ in the PS emission (Fig. S3). This shift could be attributed to the increase of perovskite grain size. ${ }^{26}$ Very interestingly, crystal grains with a size similar to the layer thickness are obtained (see Figs. 2c and 2d), which is a crucial aspect for obtaining high performance optoelectronic devices. The presence of QDs also produces an increase of the film thickness (see Fig. S4), from $210 \mathrm{~nm}$ (PS film without QDs) to $365 \mathrm{and} 420 \mathrm{~nm}$, when QDs are dispersed in PS solutions with concentrations of 300 and $400 \mathrm{mg} / \mathrm{ml}$, respectively. Independently of the thickness, very homogeneous films are obtained for the different conditions used with our novel preparation methodology. 

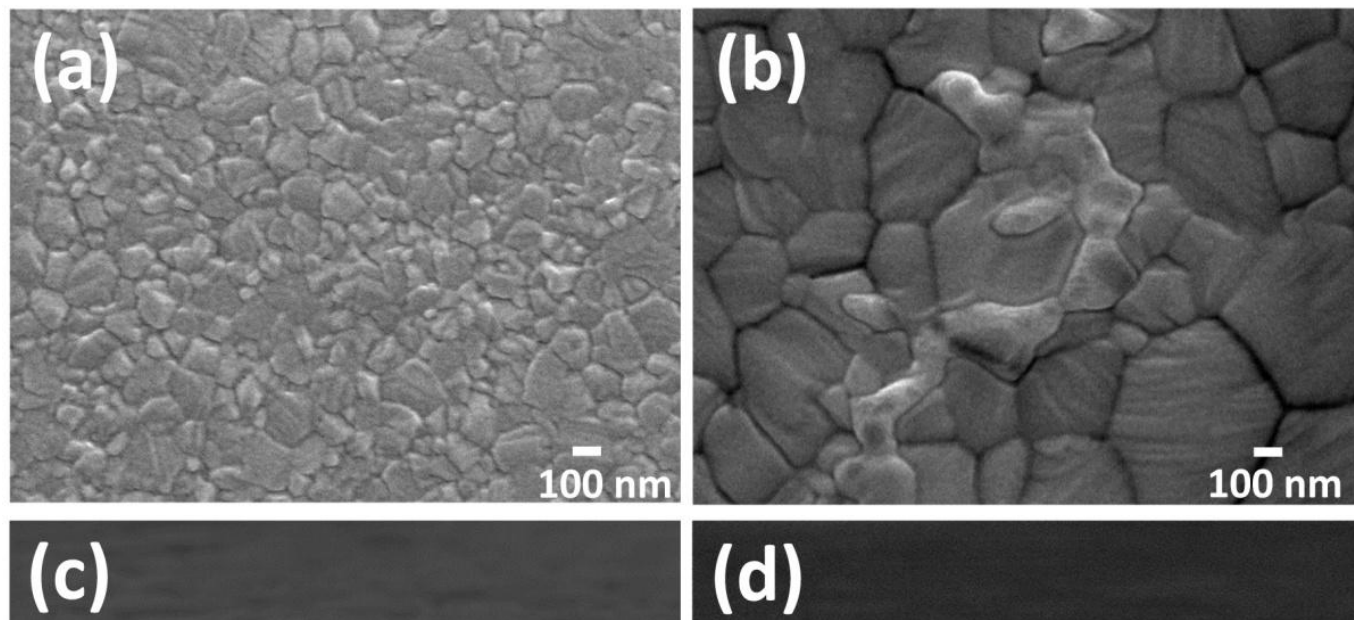

$100 \mathrm{~nm}$

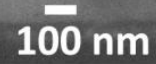

Fig 2. SEM secondary electron images of the top view of PS films (a)without and (b) with $\mathrm{PbS} / \mathrm{CdS}$ core/shell QDs $2.3 \mathrm{~nm}$ with the concentration of $300 \mathrm{mg} / \mathrm{ml}$. Cross section image of (b) sample using (c) secondary electrons and (d) backscattered electrons that allows a clearer distinction among the different layers.

Fig. 3 shows that PS layers with embedded QDs exhibit the PL emission band ascribed to the exciton recombination in the PS, but no additional QD contribution is observed within the comprised spectral range (Fig 3a). However, these composite films exhibit a strong exciplex emission $(\approx 1.02 \mathrm{eV})$ that is at a lower energies than the bandgaps of the corresponding single semiconductors themselves, as shown in Fig 3b. As we reported previously, the observed exciplex relaxation corresponds to the radiative recombination of electrons from the conduction band (CB) of PS with those holes present in the valence band (VB) of QDs. $^{14}$ 

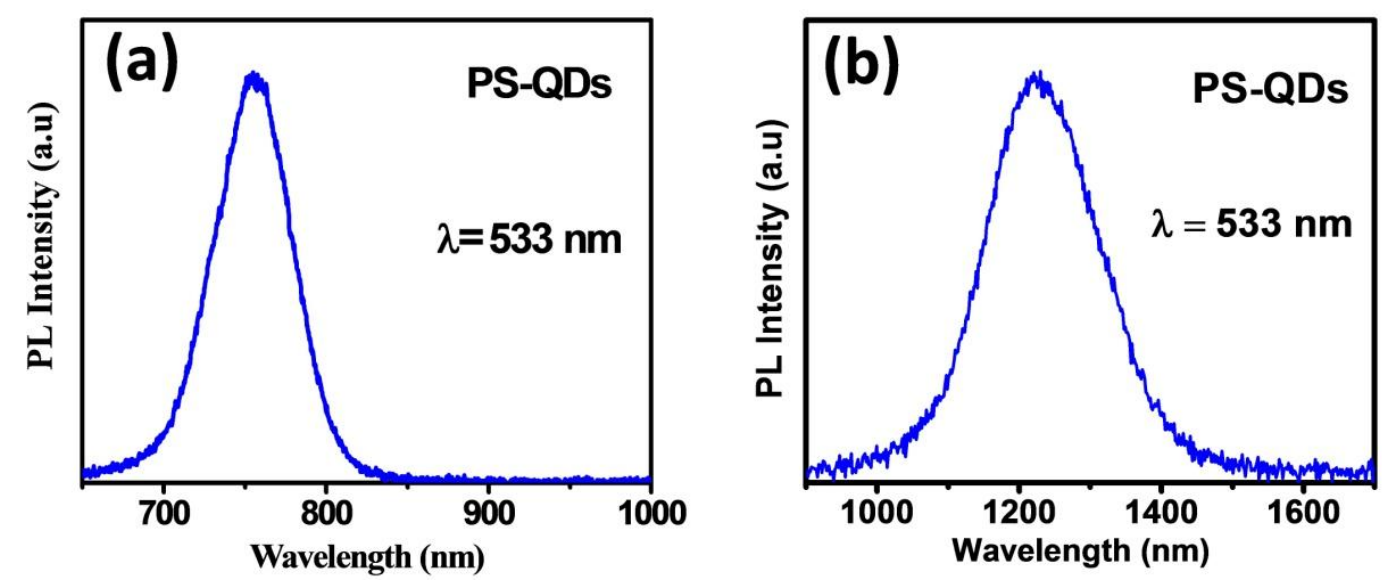

Fig 3.Room temperature PL spectra at(a) 600-1000 nm (Si photodetector) and (b) 900-1700 nm (InGaAs photodetector) wavelength ranges of PS-QDs (PbS/CdS $2.3 \mathrm{~nm}$ in diameter) film in which PS capped QDs were added in the solution of PS with a concentration of $300 \mathrm{mg} / \mathrm{ml}$. The PS-QDs films were spin coated at $9000 \mathrm{rpm}$ and annealed at $65^{\circ} \mathrm{C}$ for 1 minute and then at $100^{\circ} \mathrm{C}$ during 2 minutes.

In addition to optical pumping, electronic injection (electroluminescence, EL) was also investigated by the preparation of complete LED devices based on the configuration depicted in Fig. 4a; using a compact $\mathrm{TiO}_{2}$ layer and spiro-OMeTAD as electron and hole injecting layers, respectively. ${ }^{6}$ For those devices containing QDs, three EL emission signals that correspond to direct exciton recombination in PS and QDs (Fig. 4b) and the indirect PS-QD exciplex (Fig 4c) are observed, respectively. Note that there is a slight shift in the exciplex peak position comparing Fig. 4c with Fig. 3b, which arises from small variations of the QD size distributions from batch to batch syntheses. It is worth highlighting that the EL signal of the PS moiety of the PS-QDs composite sample is quenched by approximately one order of magnitude (see Figs. S5, S6, S7a) compare to the reference sample without embedded QDs. Moreover, QD EL intensity increases as the applied voltages increases, and this signal enhancement is even more evident at lower QD concentration (Fig. S7b). In addition, the position of QD emission was dependent on the QD concentration in the PS-QDs solution; more specifically the quenching of PS emission is expected from the type II junction, as 
already reported in our previous study based on PS-QDs bilayers (Fig. S8), ${ }^{14}$ which in turn promotes the origin of the exciplex state (Fig. 4c).

Finally, the samples prepared with embedded single core PbS QDs are compared to those devices prepared with $\mathrm{PbS} / \mathrm{CdS}$ core/shell QDs (see Fig. S9). Briefly, the OA ligands of the $\mathrm{PbS}$ core were exchanged with a PS shell, following the same procedures as for the $\mathrm{PbS} / \mathrm{CdS}$ core/shell QDs (including the concentration of these QDs in the solution of PS). In agreement with our previous work, ${ }^{27}$ the CdS shell induces a notable enhancement of the EL signal, not only from the QDs but also from the PS, as one would expect from the efficient passivation of the surface states (Fig. S9).
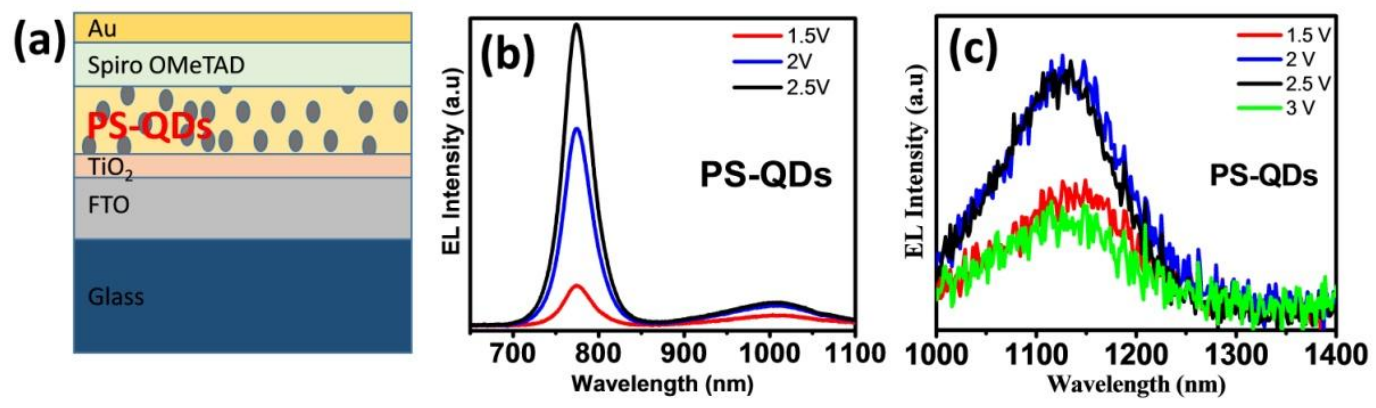

Fig 4. (a) Schematic of the LED configuration. Electroluminescence at (b) 600-1100 nm ( $\mathrm{Si}$ photodetector) and (c) 1000-1400 $\mathrm{nm}$ (InGaAs photodetector) wavelength range of PS-QDs devices at different applied voltages with $\mathrm{PbS} / \mathrm{CdS}$ core/shell $2.3 \mathrm{~nm}$ QDs $(300 \mathrm{mg} / \mathrm{ml})$ embedded in PS matrix. PS-QDs film was spin coated at $9000 \mathrm{rpm}$ then annealed at $65^{\circ} \mathrm{C}$ for 1 minute and at $100^{\circ} \mathrm{C}$ during 2 minutes.

In summary, we have developed a the new method for producing PS layers with embedded core and core/shell QDs, that yields highly homogeneous, low roughness and pinhole free PS-QDs composite films. The introduction of embedded QDs has a dramatic effect on the perovskite crystal grains, whose size is enlarged by a 4-5 fold factor, compared to the reference samples without QDs; it is worth mentioning that the crystal size measured for PS-QDs films is in the same order of magnitude than the overall layer thickness. Very importantly, the strong interaction between PS and the embedded QDs is promoting the emission 
from PS-QDs exciplex; therefore, our approach constitutes a valuable proof of concept on the coupling of these two important families of materials. In particular, exploiting our methodology and device configuration constitutes a suitable tool for producing intimately packed films, where the interaction between PS and QDs can be induced and monitored by applying both optical pumping and electrical injection. The development of high quality layers and devices that not only show optical but also electrically induced exciplex states, opens up a highly interesting scenario for the development of new and advanced optoelectronic devices; from wavelength tunable LEDs to intermediate bandgap solar cells.

\section{Acknowledgments:}

This work was supported by Generalitat Valenciana (projects PROMETEOII/2014/NNN, PROMETEOII/2014/059, and ISIC/2012/008) and Spanish MINECO (Ministry of Economy and Competitiveness) under projects MAT2013-47192-C3-1-R, MAT2015-70611-ERC and TEC2014-53727-C2-1-R). T.T.N. thanks Spanish MINECO for her scholarship.

\section{REFERENCES}

1. http://www.nrel.gov/ncpv/images/efficiency_chart.jpg

2. A. Kojima, K. Teshima, Y. Shirai and T. Miyasaka, J. Am. Chem. Soc., 2009, 131, 6050-6051.

3. W. Tress, N. Marinova, O. Inganäs, M. K. Nazeeruddin, S. M. Zakeeruddin and M. Graetzel, Adv. Energy Mater., 2015, 5, n/a-n/a.

4. D. Bi, W. Tress, M. I. Dar, P. Gao, J. Luo, C. Renevier, K. Schenk, A. Abate, F. Giordano, J.-P. Correa Baena, J.-D. Decoppet, S. M. Zakeeruddin, M. K. Nazeeruddin, M. Grätzel and A. Hagfeldt, Science Advances, 2016, 2, e1501170.

5. B. R. Sutherland and E. H. Sargent, Nat Photon, 2016, 10, 295-302.

6. O. A. Jaramillo-Quintero, R. S. Sanchez, M. Rincon and I. Mora-Sero, J. Chem. Phys. Lett., 2015, 6, 1883-1890.

7. Z.-K. Tan, R. S. Moghaddam, M. L. Lai, P. Docampo, R. Higler, F. Deschler, M. Price, A. Sadhanala, L. M. Pazos, D. Credgington, F. Hanusch, T. Bein, H. J. Snaith and R. H. Friend, Nat Nano, 2014, 9, 687-692.

8. H. Cho, S.-H. Jeong, M.-H. Park, Y.-H. Kim, C. Wolf, C.-L. Lee, J. H. Heo, A. Sadhanala, N. Myoung, S. Yoo, S. H. Im, R. H. Friend and T.-W. Lee, Science, 2015, 350, 1222-1225.

9. I. Suárez, E. J. Juárez-Pérez, J. Bisquert, I. Mora-Seró and J. P. Martínez-Pastor, Adv. Mater., 2015, 27, 6157-6162.

10. G. Xing, N. Mathews, S. S. Lim, N. Yantara, X. Liu, D. Sabba, M. Grätzel, S. Mhaisalkar and T. C. Sum, Nat Mater, 2014, 13, 476-480. 
11. F. Deschler, M. Price, S. Pathak, L. E. Klintberg, D.-D. Jarausch, R. Higler, S. Hüttner, T. Leijtens, S. D. Stranks, H. J. Snaith, M. Atatüre, R. T. Phillips and R. H. Friend, J. Chem. Phys. Lett., 2014, 5, 1421-1426.

12. H. Zhu, Y. Fu, F. Meng, X. Wu, Z. Gong, Q. Ding, M. V. Gustafsson, M. T. Trinh, S. Jin and X. Y. Zhu, Nat Mater, 2015, 14, 636-642.

13. Z. Ning, X. Gong, R. Comin, G. Walters, F. Fan, O. Voznyy, E. Yassitepe, A. Buin, S. Hoogland and E. H. Sargent, Nature, 2015, 523, 324-328.

14. R. S. Sanchez, M. S. de la Fuente, I. Suarez, G. Muñoz-Matutano, J. P. MartinezPastor and I. Mora-Sero, Science Advances, 2016, 2, e1501104.

15. P. V. Kamat, J. Phys. Chem. C, 2008, 112, 18737-18753.

16. S. A. McDonald, G. Konstantatos, S. Zhang, P. W. Cyr, E. J. D. Klem, L. Levina and E. H. Sargent, Nat Mater, 2005, 4, 138-142.

17. A. J. Nozik, M. C. Beard, J. M. Luther, M. Law, R. J. Ellingson and J. C. Johnson, Chem. Rev., 2010, 110, 6873-6890.

18. G. H. Carey, A. L. Abdelhady, Z. Ning, S. M. Thon, O. M. Bakr and E. H. Sargent, Chem. Rev., 2015.

19. Z. Yang, A. Janmohamed, X. Lan, F. P. García de Arquer, O. Voznyy, E. Yassitepe, G.-H. Kim, Z. Ning, X. Gong, R. Comin and E. H. Sargent, Nano Lett., 2015, 15, 7539-7543.

$20 . \quad$ D. N. Dirin, S. Dreyfuss, M. I. Bodnarchuk, G. Nedelcu, P. Papagiorgis, G. Itskos and M. V. Kovalenko, J. Am. Chem. Soc., 2014, 136, 6550-6553.

21. G. Seo, J. Seo, S. Ryu, W. Yin, T. K. Ahn and S. I. Seok, J. Chem. Phys. Lett., 2014, 5, 2015-2020.

22. L. Etgar, P. Gao, P. Qin, M. Graetzel and M. K. Nazeeruddin, Journal of Materials Chemistry A, 2014, 2, 11586-11590.

23. L. Hu, W. Wang, H. Liu, J. Peng, H. Cao, G. Shao, Z. Xia, W. Ma and J. Tang, Journal of Materials Chemistry A, 2015, 3, 515-518.

24. X. Gong, Z. Yang, G. Walters, R. Comin, Z. Ning, E. Beauregard, V. Adinolfi, O. Voznyy and E. H. Sargent, Nat Photon, 2016, 10, 253-257.

25. S.-S. Li, C.-H. Chang, Y.-C. Wang, C.-W. Lin, D.-Y. Wang, J.-C. Lin, C.-C. Chen, H.-S. Sheu, H.-C. Chia, W.-R. Wu, U. S. Jeng, C.-T. Liang, R. Sankar, F.C. Chou and C.-W. Chen, Energy \& Environmental Science, 2016, 9, 1282-1289.

26. V. D'Innocenzo, A. R. Srimath Kandada, M. De Bastiani, M. Gandini and A. Petrozza, Journal of the American Chemical Society, 2014, 136, 17730-17733.

27. R. S. Sanchez, E. Binetti, J. A. Torre, G. Garcia-Belmonte, M. Striccoli and I. Mora-Sero, Nanoscale, 2014, 6, 8551-8555. 